\title{
On magnetic measurements in prominences
}

\author{
I. S. Kim and O. I. Bugaenko \\ Lomonosov Moscow State University, Sternberg Astronomical institute, \\ 119992 Universitetsky pr-t, 13, Moscow, Russia \\ email: kim@sai.msu.ru
}

\begin{abstract}
The successes of magnetic measurements in faint objects located near very bright ones are strongly depending on the stray light in the telescope. We propose a mask with a variable transmission placed on the primary optics of a telescope. Our computations of the stray light in such a telescope indicate that the calculated coronagraphic factor of improvement, $K$, would increase at least by 2 orders of magnitude compared to the Lyot-type coronagraph.
\end{abstract}

Keywords. Sun:prominences, Sun: magnetic fields, techniques: polarimetric

\section{Reducing the stray light in telescopes}

In prominences the Zeeman splitting is $3-4$ orders of magnitude less than the line width. Therefore, in spite of available advanced polarimeters and recording systems, "weak" magnetic fields measurements are still complicated because of the stray light, $I_{\text {stray }}$, caused by non-object signatures in the final focal plane of a telescope. Different effects affect the measurements: optical aberrations (1), a ghost solar image produced by multiple reflections in the primary lens (2), random inhomogeneities in the glass of the primary lens (3), diffraction of the solar disk light at the entrance aperture, $I_{d i f}(4)$, scattering at micro-roughness of the primary optics, $I_{s c}(5)$, the sky brightness, $I_{s k y}(6)$, the continuum corona, $I_{\text {cont }}(7)$. The items $(2-5)$ are negligible during the totality of solar eclipses and dominate for the non-eclipse coronagraphic observations.

An ideal super-smooth primary optics is assumed below, e.g. $I_{\text {stray }}=I_{\text {dif }}$. The diffraction of a bright round source at the round-shape aperture was treated by Nagaoka (1920) and Sazanov (1968). The latter suggested the simplified expression for the range $\mathrm{R}<1.3 R_{\odot}$.

$\lg I_{d i f}(R)=\lg \frac{2 \lambda}{\pi^{3} D \gamma_{0}}+\lg \left(\frac{\sqrt{1+(\nu-1)^{2}}}{\nu-1}-\frac{\sqrt{1+(\nu+1)^{2}}}{\nu+1}\right)-0.27(\nu-1)-0.017$,

where $R$ (the distance from the solar disk center) $=\nu a, a=\frac{\pi D}{\lambda} \gamma_{0}$ is the radius of the assumed round source in arbitrary units, $D$ is the diameter of the primary lens, $\gamma_{0}$ is the angular radius of the source. Intensities are given in units of $1 \AA$ nearby the solar disk continuum. The $I_{d i f}(R)$ for $\mathrm{H} \alpha$ and near IR lines for $0.5 \mathrm{~m}$ and $4 \mathrm{~m}$ apertures, and the acceptable level of the stray light for "weak" magnetic measurements were presented recently (Kim et al. 2012). For a $0.5 \mathrm{~m}$ aperture of a non-coronagraphic telescope, $I_{d i f}$ in $\mathrm{H} \alpha$ reaches $10^{-3}$ at the prominence heights $\left(\approx 40^{\prime \prime}\right)$ and is $>5 \cdot 10^{-3}$ at the chromosphere level $\left(\approx 4^{\prime \prime}\right)$.

\subsection{Internal occulting technique (the Lyot method)}

The coronagraphic technique suggested by Lyot (1930) is based on putting a mask both in the primary focal plane and in the plane of the exit pupil to minimize the input of items $(2-4)$. The Lyot-type coronagraph has a primary single lens, a mask in the 
primary focal plane (an artificial Moon), a field lens, a mask in the plane of the exit pupil (the Lyot-stop), the relay optics, and a final focal plane. In practice, the correct use of the Lyot method results in reducing $I_{d i f}$ by $1-2$ orders of magnitude which is the coronagraphic factor, $K$, depending on the size of the mask in the primary focal plane and in the plane of the Lyot stop. For a $0.5 \mathrm{~m}$ aperture, $K \approx 20-100$ for prominences and $<20$ for the chromosphere depending on the height observed.

\subsection{Apodizing with a special mask in the plane of an entrance aperture}

The diffraction pattern in the focal plane is a result of the discontinuity of the transmission function, $G$, (or its derivatives) of the entrance aperture. $I_{d i f}$ at the optical axis is defined by $I_{d i f}(0)=1-J_{0}^{2}(a)-J_{1}^{2}(a)$, where $J_{1}(x)$ is the Bessel function of the first kind, $a=\left(\pi D \sin \gamma_{0}\right) / \lambda, \lambda$ is the wavelength, $\gamma_{0}$ is the angular radius of solar disk, and $D$ is the diameter of the aperture. If $a \gg 1$, then $I_{d i f}(0) \approx 1-2 /(\pi a)$ (Kim et al. 1995). For $\gamma_{0}=960^{\prime \prime}, \lambda=600 \mathrm{~nm}, D=200 \mathrm{~mm}$, we obtain $a=4874$ and $\left.I_{\text {dif }}(0) \approx 1.3\right) \cdot 10^{-4}$. A mask with variable transmission $G(\rho)=1-\rho^{2}$ has discontinuities in the first derivative. For $\gamma=(1+\epsilon) \gamma_{0}$, the apodized $\left(I_{\text {dif }}^{a}\right)$ and non-apodized $\left(I_{d i f}\right)$ are related as following:

$$
I_{d i f}^{a}(\epsilon) \simeq 2 / 3 \pi^{4}\left[I_{d i f}(\epsilon)\right]^{3} .
$$

Let's estimate the $K$ factor for the chromosphere and prominence heights and $a \approx 5000$.

- Quiescent prominence: $h=40^{\prime \prime}, \epsilon=0.04\left(\epsilon \gamma_{0}=40^{\prime \prime}\right)$. Then $I_{\text {dif }}(0.04)=10^{-3}$, $I_{\text {dif }}^{(a)}(0.04)=0.7 \cdot 10^{-7}$, and $K=10^{4}$.

- The upper chromosphere: $h=4^{\prime \prime}, \epsilon=0.004\left(\epsilon \gamma_{0}=4^{\prime \prime}\right)$. Then $I_{d i f}(0.004)=10^{-2}$, $I_{\text {dif }}^{a}\left(\overline{0.004)}=0.7 \cdot 10^{-4}\right.$, and $K=10^{2}$.

We note that the mask $\left(1-\rho^{2}\right)$ reduces the output by 3 times.

Summary. Current state of technology suggests to put on the primary optics a mask with a variable transmission $G(\rho)=1-\rho^{2}$ for having a low-scattered light telescope. At prominence heights, the calculated coronagraphic factor, $K$, is at least 2 orders of magnitude higher as compared with the Lyot-type coronagraph.

Acknowledgements. The reported study was partially supported by RFBR (research project No. 11-02-00631), IAU, SCOSTEP, SF2A and KLSA/CAS.

\section{References}

Kim, I. S., Bugaenko, O. I., \& Bruevich, V. V. and Evseev, O. A., 1995, Bull. of Rus. Acad. of Sci., 59, 153.

Kim, I. S, Alexeeva, I. V, \& Suyunova, E. Z., 2012, ASP Conference Series, T. R. Rimmele, M. Collados Vera et al., eds, Vol. 463, 337.

Lyot, B., Comptes Rendus Acad. Sci., 1931, 1169.

Nagaoka, H., 1920 Astrophysical Journal, 51, 73.

Sazanov, A. A., 1968, Ph.D. thesis, IZMIRAN, Moscow. 